\title{
Aspiraciones y expectativas de las familias de ascendencia extranjera en el País Vasco: educación, formación, trabajo y movilidad social
}

\author{
Aspirations and expectations of families of foreign \\ descent in the Basque Country: education, training, \\ work and social mobility \\ Iraide Fernández Aragón \\ Universidad del País Vasco / Euskal Herriko Unibertsitatea \\ iraide.fernandez@ehu.eus \\ Julia Shershneva \\ Universidad del País Vasco / Euskal Herriko Unibertsitatea \\ yulia.shershneva@ehu.eus \\ Maite Fouassier Zamalloa \\ Ikuspegi. Observatorio Vasco de Inmigración \\ maite.fouassier@ehu.eus
}

Cómo citar/Citation: Fernández, Iraide, Julia Shershneva y Maite Fouassier. 2020. «Aspiraciones y expectativas de las familias de ascendencia extranjera en el País Vasco: educación, formación, trabajo y movilidadsocial». Deusto JournalofHuman Rights, No. 6:179-210. doi: http://dx.doi.org/10. 18543/djhr.1900.

Sumario: Introducción. 1. Factores determinantes de las expectativas educativas y formativas en el alumnado de ascendencia extranjera. 2. Metodología. 3. Análisis de resultados. 3.1. Exploración cuantitativa. 3.2. Expectativas y aspiraciones desde la perspectiva de las familias. Conclusiones. Referencias bibliográficas.

Resumen: En este artículo se presentan los principales resultados de un estudio sobre los hijos e hijas de la inmigración en el País Vasco. Concretamente nos detenemos en las aspiraciones y expectativas que se generan en el seno familiar, tanto desde el punto de vista de los progenitores como desde sus descendientes con respecto a su futuro. A partir de una metodología mixta, abordamos las opiniones y percepciones de los miembros de las familias de ascendencia extranjera en relación a aquellos elementos que intervienen en una trayectoria educativa encaminada al éxito como garante de una movilidad social 
ascendente. El análisis muestra que el capital social y cultural son considerados por las familias elementos para lograr una mayor integración económica y social. Los progenitores afirman por medio de sus discursos la importancia de la educación, el conocimiento del euskera y una red social fuerte compuesta por personas autóctonas.

Palabras clave: expectativas, capital social, inmigración, familia, educación.

Abstract: This article presents the main results of a study on the sons and daughters of immigrants in the Basque Country. Specifically, we look at the aspirations and expectations generated within the family, both from the point of view of the parents and their descendants with regard to their future. Using a mixed methodology, we address the opinions and perceptions of family members of foreign descent in relation to those elements involved in an educational path aimed at success as a guarantee of upward social mobility. The analysis shows that social and cultural capital are considered elements for achieving greater economic and social integration. Through their speeches, parents affirm the importance of education, knowledge of the Basque language and a strong social network made up of indigenous people.

Keywords: expectations, social capital, immigration, family, education. 


\section{Introducción}

Las aspiraciones de las y los jóvenes y sus progenitores ante su futuro es una cuestión que inquieta, en mayor o menor medida, a todas las familias. Si, como es el caso que nos ocupa, hablamos de población de ascendencia extranjera, la realidad nos lleva a tomar en consideración una serie de variables que contribuyen a dibujar diversos escenarios en el seno familiar. Veremos en qué contextos, bajo qué indicadores, si se dan diferencias entre el posicionamiento ante su propio futuro y si coincide con el de sus progenitores, o si aparecen otros elementos que actúan en las expectativas generadas por todos los integrantes de la institución familiar.

Este artículo emana de una investigación mucho más amplia que pretende realizar un primer acercamiento al estudio y análisis de los hijos e hijas de las personas de origen extranjero en el País Vasco (Oleaga 2017). La literatura internacional lleva décadas recogiendo esta realidad, como los textos de Rumbaut (1994, 2004), Portes (1996), y este junto con Zhou (1993), Kao y Tienda (1998) y Majoribanks (2003), entre otros. A nivel estatal ya contábamos con estudios de temática similar, como los trabajos de Aparicio y Tornos (2006) sobre hijos de inmigrantes, la investigación longitudinal de la segunda generación liderada por Aparicio y Portes (2014) donde se examinan las trayectorias educativas y laborales de los hijos e hijas de las personas extranjeras venidas a España, su identificación cultural, experiencias de discriminación y aspiraciones de futuro, o el estudio longitudinal y comparativo centrado en el abandono escolar en varios países europeos entre los que se encuentra España y que presta una atención especial a las aspiraciones y el apoyo familiar y del profesorado (Carrasco, Narciso y Bertran-Tarrés 2018). También contamos con investigaciones sobre jóvenes de ascendencia extranjera limitadas a las comunidades autónomas (Portes, Aparicio y Haller 2009, Checa y Arjona 2009, Gualda 2010). En el caso concreto del País Vasco, tenemos como referencia el trabajo que se ha elaborado desde Ikuspegi (Oleaga 2017), donde se realiza un análisis pormenorizado de las y los hijos de la inmigración en Euskadi, que incluye las miradas desde el ámbito familiar, educativo y comunitario. Sin embargo, los estudios que encontramos en relación con la infancia y la juventud de origen extranjero son, en su mayoría, desde el campo de la educación, como el trabajo de Intxausti, Etxeberria y Joaristi (2014) sobre las expectativas del futuro de las familias inmigrantes y el profesorado en el País Vasco, los análisis llevados a cabo sobre integración y alumnado inmigrante (Vicente Torrado 2006, 2008, Etxeberria y Elosegui 2010a, 2010b), o sobre la diversidad en la escuela (Barquín 2007, 2015). 
En nuestro caso, cuando nos referimos a familias de ascendencia extranjera buscamos incluir a todas aquellas personas que han migrado desde sus países de origen, a las que se han nacionalizado, aquellas que ya han nacido en el Estado y a quienes aún naciendo en este territorio mantienen la nacionalidad de origen de sus progenitores. Según los datos del Instituto Nacional de Estadística y de las concesiones de nacionalidad emitido por el Ministerio de Inclusión, Seguridad Social y Migraciones, hemos estimado que, en 2019 , un 10,8\% de la población en el País Vasco tiene ascendencia extranjera, dato que aumenta al 15,4\% a nivel estatal.

Los únicos datos disponibles sobre la realidad migratoria en el contexto familiar en la Comunidad Autónoma de Euskadi (CAE), indican que, según el Censo 2011, un 15,9\% de los jóvenes de hasta 24 años tiene al menos un progenitor de nacionalidad $u$ origen extranjero, que asciende a un 17,9\% si atendemos a los menores de 5 años (Otero y Mendoza 2017).

A lo largo de este texto abordaremos, por una parte, las aspiraciones educativas y formativas que los propios jóvenes dejan asomar por medio de las respuestas en relación a lo que ellos consideran importante para su futuro, contrastando con la opinión que expresan sus progenitores ante las aspiraciones que tienen para sus hijos e hijas. Por otra parte, damos espacio a sus expectativas, donde se rebaja ese grado de deseabilidad, dando paso a lógicas más racionales ante la posibilidad de conseguir un determinado objetivo vital.

Para abordar las expectativas y aspiraciones educativas, formativas y ocupacionales, tanto de los hijos e hijas como de los progenitores, contamos con los resultados de un sondeo probabilístico a personas autóctonas sin ascendencia extranjera y a hijos e hijas de ascendencia extranjera en el País Vasco. Como complemento a este estudio de corte cuantitativo, se analiza el contenido de los discursos de familias de ascendencia extranjera, que nos proporcionan gran información, matiza y profundiza en las variables analizadas anteriormente. El propósito no es otro que realizar una primera aproximación a un fenómeno social complejo.

\section{Factores determinantes de las expectativas educativas y formativas en el alumnado de ascendencia extranjera}

Las expectativas de las y los jóvenes y sus familias suelen abordarse desde el punto de vista educativo. Es decir, el alcance de ciertas metas futuras suele ponerse en relación con el rendimiento educativo (Sewell, Haller y Portes 1969), así como la fuerza e implicación que ejerce la institución familiar en tal rendimiento, que suele ser incluso mayor que 
la de la propia escuela, ya que mediante el proceso de socialización se transmiten valores, creencias, costumbres y aspiraciones educativas (Coleman 1968, Bourdieu 1977). En algunos estudios se constata que, en general, tal influencia familiar suele ser positiva para su rendimiento escolar (González-Pineda et al. 1997).

$\mathrm{Si}$ atendemos al origen cultural de estas familias, uno de los objetivos del proyecto migratorio es mejorar su vida y su posición social, y transmiten sus expectativas a sus hijos e hijas para poder no solo ofrecer sino también modelar un futuro mejor para ellos. Consideran su educación una inversión, de tal forma que la adquisición de capital cultural repercutirá en mayor capital económico (Bourdieu 1986) y, por lo tanto, promoverá una movilidad ascendente. Es decir, las familias confían en los centros escolares como garantes de una integración exitosa de sus hijos e hijas que les aportará el capital social y cultural necesario para su ascenso social. Qué itinerario inicial sea elegido para estos jóvenes dependerá igualmente de lo que aporten sus progenitores en forma de capital humano, estructuras familiares y su situación administrativa en destino, entre otras cuestiones.

Contamos, por un lado, con las propias experiencias, conocimientos y acciones formativas de los jóvenes en su desarrollo educativo que obtienen de sus familias. A mayor capital cultural, mayor formación adquirida (Bourdieu 1979). Por otra parte, el nivel socioeconómico de las familias es igualmente otro indicador de mayores posibilidades educativas $y$, por lo tanto, repercutirá en las expectativas hacia su futuro, no solo formativo, sino también laboral. Una situación socioeconómica alta en el núcleo familiar permite contar con la posibilidad de invertir en formación al mismo tiempo que las y los descendientes lograrían ser más competitivos gracias a su capital cultural. Todos estos activos sociales favorecen la movilidad social más allá de los medios económicos (Lozano y Trinidad 2019). Por último, contamos con el apoyo familiar hacia la educación de sus descendientes o lo que comúnmente se entiende como capital social. Es decir, la capacidad de la persona para poder alcanzar recursos mediante su inserción en redes o en otras estructuras sociales (Portes 2007).

Si bien este concepto aparece en los estudios de ciencias sociales de Bourdieu y Coleman, fue Hanifan (1916) quien acuñó por primera vez el término capital social en un intento por desarrollar la relevancia de la participación comunitaria en el espacio escolar. Sin embargo, su trabajo no caló con suficiente fuerza como para que estudios posteriores siguieran esta estela. Según Hanifan $(1916,134)$ «Aquellos componentes tangibles [que] cuentan sumamente en la vida diaria de las personas, a saber, la buena voluntad, la camaradería, la comprensión y el trato social entre individuos y familias, características constitutivas de la unidad social (...) 
abandonado a sí mismo, el individuo es socialmente un ser indefenso (...) pero si entra en contacto con sus vecinos, y estos con nuevos vecinos, se producirá una acumulación de capital social que podrá satisfacer de inmediato sus necesidades sociales y producir unas posibilidades sociales suficientes para mejorar de forma sustancial las condiciones de vida de toda comunidad». El capital social familiar va más allá de lo que puede considerarse las relaciones familiares, proporcionando un mejor rendimiento escolar gracias a que tales vínculos familiares permiten acumular más capital social (Torio, Hernández y Peña 2007).

La influencia que ejercen las familias inmigrantes en el rendimiento escolar de sus hijos e hijas descansa en las expectativas generadas en su proyecto migratorio. Las estrategias familiares están condicionadas por las aspiraciones que se generan hacia la educación de sus descendientes y, por tanto, sus expectativas y la función de la escuela (Garreta 1994).

De igual modo, se percibe que trayectorias educativas exitosas facilitan que las expectativas se mantengan, si bien el fracaso escolar genera un cambio en las mismas que afecta al proyecto migratorio familiar que llevó en un primer momento a tomar la decisión de desplazarse a otro país. A este respecto, Carrasco, Pamiès y Bertran (2009) consideran que se debe adoptar una perspectiva procesual para comprender las expectativas que tienen las familias.

Según Cebolla y Martínez de Lizarrondo (2015) en las expectativas educativas no influyen tanto las características del centro escolar, como puede ser la concentración de alumnado inmigrante o el nivel socioeconómico del centro, sino que atiende en mayor medida a razones individuales o familiares. Esta afirmación no está exenta de cierta controversia ya que otras investigaciones - que abarcaban a todo el alumnado y no solo al de ascendencia extranjera - afirman justamente que el nivel socioeconómico medio del centro educativo influye en el rendimiento educativo del alumnado, en mayor medida incluso que el influjo que pueda tener la familia (Lizasoain et al. 2007, Torrents et al. 2018). En todo caso, tanto la escuela como la familia son elementos de adscripción y de logro de status. Por ello, pueden o generar mayor desigualdad o ayudar a la movilidad social (Gil del Pino y García Segura 2019). Del mismo modo que se comprueba que las motivaciones educativas de la juventud de ascendencia extranjera se dirigen no solo al rendimiento, sino a la valoración social y a la recompensa social (Alcalde y Hernández 2018).

Es interesante igualmente recoger una de las conclusiones del estudio longitudinal sobre las segundas generaciones en España (Portes et al. 2011) en relación a las expectativas de la juventud y es que sitúa a la influencia familiar como un indicador causal más, entrando en 
juego la ambición temprana de los propios jóvenes como un predictor importante del logro futuro. Este mismo estudio puntualiza que el sexo interviene de forma directa, de tal forma que se percibe que las chicas presentan una mayor ambición.

Señalamos, por último, a la figura del profesorado como otro de los elementos que interviene en el rendimiento de su alumnado $y$, por lo tanto, en sus expectativas. El profesorado es un agente activo de la institución educativa que, por medio del desarrollo de sus competencias, contribuye como una pieza más al éxito o fracaso escolar, a la productividad y al consiguiente logro educativo. Algunas investigaciones afirman que el profesorado se inclina por otorgar al alumnado con un alto nivel socioeconómico unas características que le conducirán al éxito escolar, como es un mejor rendimiento y mayores capacidades para el estudio (Van Houtte 2011). Es decir, bajo criterios de clase social se genera una imagen de estudiante ideal. La juventud, por su parte, percibe que el profesorado genera unas expectativas más bajas sobre ellos que sus familias (Carrasco, Pàmies y Narciso 2018). El sentimiento de apoyo por parte del profesorado es necesario para poder eliminar esta percepción que, si se mantiene, puede acabar contribuyendo a una menor vinculación con la escuela y, por lo tanto, menor confianza en sus propias capacidades y en el sistema educativo.

Ante todos estos elementos definitorios que hemos desarrollado en este apartado, comenzaremos a ver a continuación en qué medida todos ellos intervienen en las expectativas de las y los jóvenes de ascendencia extranjera.

\section{Metodología}

Para acercarse a la realidad de la descendencia de las personas de origen extranjero se ha optado por una estrategia metodológica mixta. Por un lado, en una primera fase se optó por una aproximación cuantitativa mediante la técnica de encuesta, y, por otro lado, se realizó una aproximación cualitativa utilizando las entrevistas grupales a familias.

En la primera fase, se realizaron 323 encuestas al alumnado de primaria y secundaria de entre 6 y 16 años y sus progenitores o tutores. Para ello se realizó un sondeo probabilístico a una muestra representativa de nuestro público objetivo compuesto por 223 encuestas a hijos/as de personas de origen extranjero y sus progenitores y 100 a personas autóctonas (sin ascendencia extranjera), con afijación proporcional, teniendo en cuenta el origen de los progenitores, el curso (primaria/ secundaria) y las cuotas de edad (cuatro franjas: 6-7 años, 9-10 años, 
12-13 años y 15-16 años, correspondientes a los siguientes niveles educativos: $1 .^{\circ}$ primaria, $4 .^{\circ}$ primaria, $1 .^{\circ}$ secundaria, $4 .^{\circ}$ secundaria). La selección de las personas encuestadas se realizó aleatoriamente, con encuestación presencial a pie de calle y 39 puntos de muestreo. Con este tamaño muestral, nos garantizamos trabajar con un error muestral máximo del $+/-5,44 \%$ y con un nivel de confianza estadístico del 95\% (para el supuesto más desfavorable, donde $p=q=0,5$ ).

Tabla 1

Distribución de la muestra ponderada

\begin{tabular}{|l|c|c|}
\hline & Primaria & Secundaria \\
\hline España & 30,4 & 31,6 \\
\hline América Latina & 31,4 & 31,6 \\
\hline Magreb & 12,1 & 14,5 \\
\hline África Subsahariana & 8,7 & 6,8 \\
\hline Europa Oriental & 1,9 & 5,1 \\
\hline China & 10,7 & 1,3 \\
\hline Resto de Asia & 0,8 & 3,8 \\
\hline Otros & 4,0 & 5,1 \\
\hline Total $(\%)$ & 100 & 100 \\
\hline Total $(n)$ & 206 & 117 \\
\hline
\end{tabular}

Fuente: Elaboración propia.

En este sentido, para poder recoger la información del alumnado de primaria de edades más tempranas se confeccionó un cuestionario ilustrado que recogía las mismas dimensiones que el del alumnado más mayor, a saber, la identidad, el bienestar subjetivo, el ámbito escolar, las relaciones familiares y las expectativas futuras de progenitores y descendencia.

Por último, las encuestas se realizaron siempre en presencia de los progenitores o tutores y tutoras de los y las chicas. La recogida de la información se realizó entre marzo y junio de 2016.

Por otro lado, en una segunda fase, se llevaron a cabo 20 entrevistas grupales a las familias de origen extranjero con el objetivo de contrastar 
la información obtenida en la primera fase, así como indagar en las motivaciones y aspiraciones de las familias. Además, una entrevista familiar permite no solo conocer el discurso individual de cada miembro de la familia sino también da lugar a diálogos intergeneracionales muy interesantes. La información recogida ha sido analizada mediante la técnica de análisis de discurso.

Tabla 2

Muestra entrevistas familiares

\begin{tabular}{|c|c|}
\hline Variable & Descripción \\
\hline \multirow{5}{*}{ Origen } & 4 familias de origen chino \\
\hline & 4 familias de origen latinoamericano \\
\hline & 4 familias de origen rumano \\
\hline & 4 familias de origen magrebí \\
\hline & 4 familias procedentes de África subsahariana \\
\hline \multirow{3}{*}{ Territorio Histórico } & 7 familias de Araba \\
\hline & 6 familias de Gipuzkoa \\
\hline & 7 familias de Bizkaia \\
\hline \multirow{3}{*}{ Tipo de familia } & Monoparental \\
\hline & Nuclear \\
\hline & Extensa \\
\hline \multirow{2}{*}{ Composición familiar } & Mixta \\
\hline & Progenitores de un mismo origen \\
\hline
\end{tabular}

Fuente: Elaboración propia.

Así, la muestra discrecional se compone de 20 familias de diferentes orígenes residentes en el País Vasco, teniendo en cuenta variables como origen (China, América Latina, Rumanía, Magreb, África Subsahariana), sexo, tipo de familia (monoparental, nuclear, extensa) y territorio histórico (Álava, Gipuzkoa y Bizkaia). La contactación con las personas que participaron en la investigación se ha realizado en colaboración con una empresa experta en investigación social. Por último, la recogida de la información se realizó entre junio de 2018 y septiembre de 2019. 


\section{Análisis de resultados}

Como ya se ha mencionado en los apartados anteriores, las expectativas de los y las progenitoras, referentes y tutores/as son fundamentales en la comprensión del fenómeno de las llamadas segundas generaciones $y$, en concreto, en su propia formación de expectativas. La actitud de progenitores - que a menudo se traduce en expectativas, niveles de aspiración, interés por los estudios o ambición laboral- es crucial en la propia generación de planes futuros y cosmovisión del alumnado de ascendencia extranjera (Garreta 1994). En este sentido, la educación aparece en los estudios sociológicos como la piedra angular desde la que articular esta integración exitosa, la base sobre la que construir las mencionadas expectativas. Precisamente por este motivo, al hablar de expectativas vitales de progenitores y descendientes es condición sine qua non analizar la variable educativa. Este apartado abordará estas cuestiones desde dos puntos de vista —el de los y las progenitoras y el de la descendencia - y desde dos perspectivas metodológicas complementarias - la cuantitativa a través de la encuesta, y la cualitativa a través de las entrevistas familiares - , para lograr una imagen final de las expectativas de futuro de las personas de ascendencia extranjera en Euskadi.

\subsection{Exploración cuantitativa}

El análisis de las expectativas se realizará en primer lugar desde la perspectiva cuantitativa del fenómeno a través del análisis de la encuesta realizada a personas de ascendencia extranjera. Se trata de una encuesta compleja, realizada a progenitores y descendientes. En el caso de la descendencia menor de 12 años, es decir, cursando primaria, la encuesta contenía menos ítems y era ilustrada, para facilitar su comprensión. Esta característica técnica nos obliga a realizar un análisis de los datos por separado, aunque coincidente en sus grandes dimensiones. Es decir, si bien se preguntaba por la misma cuestión a alumnado de primaria y secundaria, las categorías de respuesta en primaria eran resumidas (e ilustradas), mientras que las de secundaria permitían más detalle y profundidad. Este hecho provoca que preguntas con diferentes categorías de respuesta y planteamientos no sean directamente comparables.

\subsubsection{ASPIRACIONES Y EXPECTATIVAS EN EDUCACIÓN PRIMARIA}

Reparamos en primer lugar en sus aspiraciones, es decir, qué quieren ser de mayor. Presentamos al alumnado encuestado una lista 
con nueve profesiones y la opción de poder decir aquella que quisiera y que no estuviera en la lista. Como muestra el gráfico 1, la opción más elegida es ser futbolista $(22,6 \%)$, seguida de policía $(21,6 \%)$. Es destacable que el $12,3 \%$ del alumnado de origen extranjero de primaria encuestado no sabe lo que quiere ser. También se escogen de manera destacable las profesiones de profesor/a (8\%), bombero/a (8\%), cocinero/a (8\%), y médica/o $(7,1 \%)$.

\section{Gráfico 1}

\section{Aspiración ser de mayor alumnado de ascendencia extranjera}

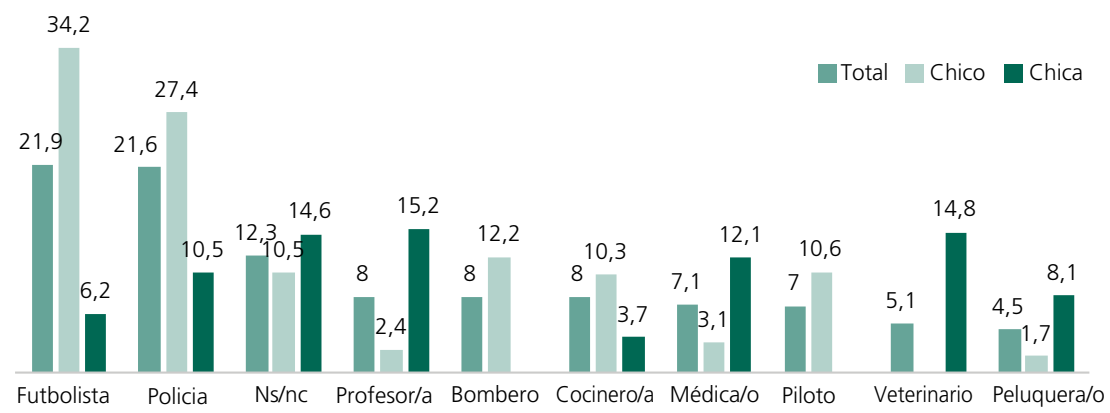

Fuente: Elaboración propia. P30. Cuando seas mayor, ¿qué te gustaría ser?

Observamos diferencias significativas al realizar cruces con la variable sexo, ya que más chicos desean ser futbolistas (34,2\%) que chicas $(6,2 \%)$, si bien hay más chicas que quieren ser profesoras $(15,2 \%)$ que chicos (2,4\%). También se dan diferencias significativas en profesiones tradicionalmente masculinizadas como bombero, policía o piloto - donde encontramos muchos más chicos- y aquellas que han sido tradicionalmente feminizadas o se vinculan a cuidados, como peluquería o veterinaria - donde encontramos un mayor porcentaje de chicas-.

En cuanto a las aspiraciones de los progenitores de origen extranjero una vez acaben de estudiar en el instituto, prácticamente ocho de cada diez familias desean que sus hijos e hijas cursen estudios universitarios (gráfico 2), mientras que un 3\% prefiere que estudien una formación profesional y un $2 \%$ que inicien su trayectoria profesional. Encontramos diferencias significativas entre el alumnado que acude a un centro público o privado/concertado. Los progenitores de centros concertados desean en mayor medida que sus hijos e hijas acudan a la universidad (86\%, frente a $80 \%$ ). Ante el porqué de sus afirmaciones sobre el interés que 
tienen porque sigan estudiando aparecen aspectos relacionados con la deseabilidad de que puedan tener un mejor empleo, un mejor futuro en su vida y porque cubrirían en algunos casos las expectativas que tienen sobre sus descendientes. El análisis de las aspiraciones familiares es tan importante como la su descendencia, o si acaso más. Tal y como mencionábamos en el marco teórico, la implicación de la familia en el proceso escolar es crucial en el rendimiento y éxito académico, incluso más que la propia escuela, ya que se trasmiten dichas aspiraciones a través del proceso de socialización (Coleman 1968). Así, podría concluirse a la luz de los resultados que, si progenitores de centros concertados desean en mayor medida que su descendencia curse estudios universitarios, esta tendrá más posibilidades de hacerlo que la que acude a un centro público, generando así una evidente desigualdad en sus trayectorias futuras.

Gráfico 2

\section{Aspiraciones de los progenitores de primaria después del instituto}

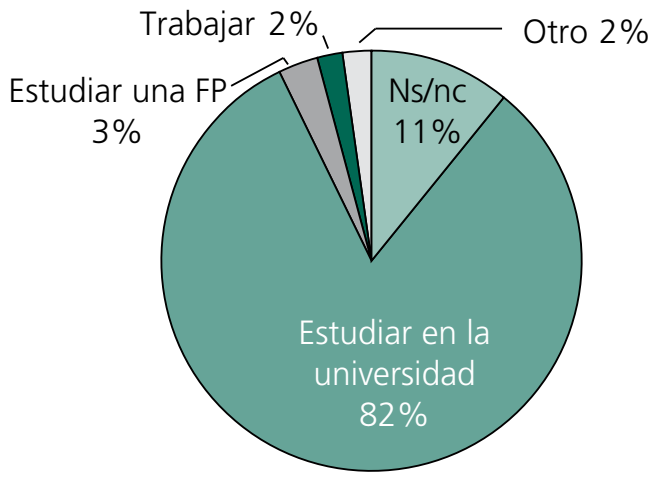

Fuente: Elaboración propia. P72. ¿Qué le gustaría que haga su hijo/a después de acabar el instituto?

Posteriormente les preguntamos de qué les gustaría que trabajaran sus hijos e hijas en una pregunta abierta, es decir, no se sugería ningún tipo de profesión como cuando preguntábamos directamente al alumnado de primaria. Una vez contestada, preguntamos realmente de qué cree que podría trabajar, comparando así la diferencia entre sus aspiraciones y las expectativas que vuelcan sobre sus hijos e hijas. Observamos en la tabla 3 que, primeramente, no saben qué contestar (30,5\%), incluso en mayor medida cuando les apelamos a ser realistas (38,8\%). Por otra parte, el deseo de que trabajen en lo que más les guste o les pueda motivar supone un $15 \%$, pero cae hasta un 5,9\% cuando el «querer» se convierte 
en «poder». Por lo tanto, los progenitores asumen que no será tan fácil que sus hijos e hijas vayan a poder trabajar de lo que quieren, al igual que vayan a poder trabajar de lo que estudien con una diferencia de 2,7 puntos de lo que desean que ocurra $(3,7 \%)$ a lo que realmente puede llegar a pasar (1\%).

Tabla 3

\section{Aspiraciones y expectativas de los progenitores de primaria sobre el futuro profesional de sus hijos e hijas}

\begin{tabular}{|c|c|c|}
\hline & Deseo & Siendo realista \\
\hline $\mathrm{Ns} / \mathrm{nc}$ & 30,5 & 38,8 \\
\hline Médico/a y sanitario/a & 18,8 & 13,1 \\
\hline Lo que le guste/quiera & 15,0 & 5,9 \\
\hline Profesor/a & 4,9 & 5,9 \\
\hline Futbolista & 4,0 & 5,8 \\
\hline De lo que estudie & 3,7 & 1,0 \\
\hline Policia & 3,5 & 2,6 \\
\hline Ingeniero/a & 3,2 & 0,9 \\
\hline Administrativa/o & 2,7 & 1,3 \\
\hline Veterinario/a & 1,4 & 3,3 \\
\hline Abogada/o & 1,4 & 0,5 \\
\hline Mecánico/a & 1,4 & 0,9 \\
\hline Arquitecto/a & 1,4 & 1,8 \\
\hline Economista & 1,0 & 1,5 \\
\hline Esteticista y Peluquería & 0,9 & 1,0 \\
\hline Informática & 0,5 & 3,2 \\
\hline En lo que pueda & 0,5 & 0,9 \\
\hline Artista & 0,4 & - \\
\hline
\end{tabular}

Fuente: Elaboración propia. P74. ¿De qué le gustaría que trabaje su hijo/s de mayor? P75. Y siendo realista, ¿de qué cree que podrá trabajar? 
En el caso de profesiones concretas, observamos que hay quienes apuestan más por el deseo que por lo que realmente creen que vaya a ocurrir -ingenierías, rama sanitaria-, y quienes creen que su futuro está más ligado a dónde pueden conseguir un empleo que al deseo de que trabajen en ello —informática, veterinario/a-.

\subsubsection{AspiRACIONES Y EXPECTATIVAS EN EDUCACIÓN SECUNDARIA}

Al igual que entre el alumnado de primaria, se abordaron las aspiraciones y expectativas del alumnado de ascendencia extranjera de secundaria sobre su futuro académico y laboral, así como las que mantienen sus progenitores. Como se aprecia en el gráfico 3 , tras finalizar la enseñanza obligatoria, un $60,8 \%$ del alumnado de ascendencia extranjera desea ir a la universidad y un $27,7 \%$ quiere estudiar una formación profesional. Es decir, un 88,5\% tiene intención de continuar con sus estudios. Tan solo un 1,9\% se ve en el mercado laboral. Concretamente, y atendiendo a las variables de cruce significativas, los chicos se ven en mayor medida estudiando una formación profesional $(43,5 \%)$ frente a las chicas $(7,5 \%)$ y, en cambio, las chicas desean ir en mayor medida a la universidad $(77,5 \%$ frente al $47,7 \%$ ).

\section{Gráfico 3}

\section{Aspiraciones del alumnado de secundaria una vez finalizado el instituto}

Total Chicos $\square$ Chicas

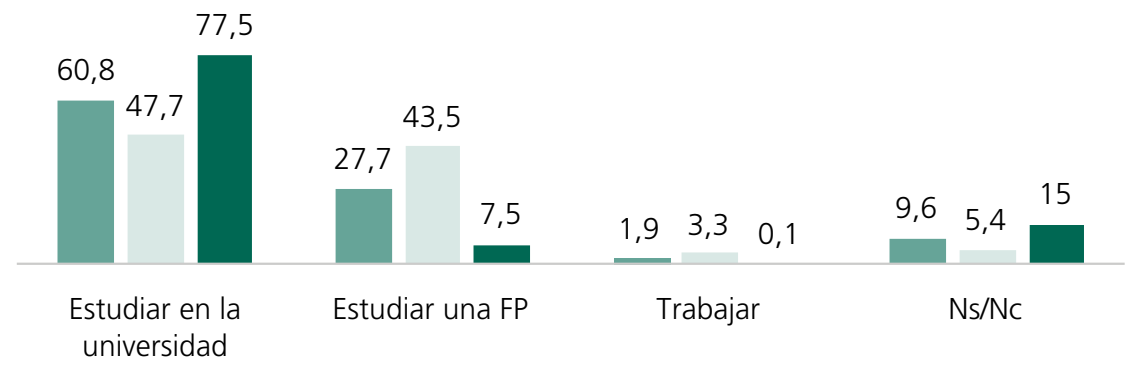

Fuente: Elaboración propia. P55. ¿Qué te gustaría hacer después de acabar el instituto? 
Resulta también interesante el análisis de estas respuestas en función del modelo lingüístico y el origen de la ascendencia. Respecto al primero, un $50 \%$ de estudiantes de modelo A se ven trabajando una vez acabado el instituto, diferenciándose significativamente del resto de modelos que no contemplan tal opción. En lo referente al origen o ascendencia, es relevante para comprender las aspiraciones del alumnado de secundaria. Aquellos alumnos con ascendencia asiática optan en mayor medida por el mundo laboral tras terminar la enseñanza obligatoria (un 25\%), muy por encima de la media (1,9\%), también por encima de la media del alumnado que desea estudiar formación profesional se encuentra el de ascendencia de Europa del este.

Las aspiraciones de las personas de referencia, ya sea familia o profesorado, pueden ser relevantes o incidir también en las propias expectativas del alumnado. Al preguntarles por la idea que creen que tienen sus progenitores y el profesorado sobre su propio futuro, vemos en el gráfico 4 que tienen una percepción algo diferente a la que ellos han expresado en el caso de su familia, y aún más alejada de la del profesorado. El 52\% del alumnado de ascendencia extranjera de secundaria cree que sus progenitores desean que vayan a la universidad, en menor medida que lo que ellos piensan sobre su propio futuro. Sí que coincide, sin embargo, la idea de estudiar una formación profesional $(27,7 \%)$ y aumenta levemente el desconocimiento sobre lo que piensa su familia (14,9\%). En el caso del profesorado, un $49,8 \%$ ignora lo que pueden pensar sobre su futuro, un $40,5 \%$ cree que el centro escolar piensa de él que va a continuar sus estudios en la universidad. La formación profesional desciende a un 6,3\%. 
Gráfico 4

\section{Percepción del alumnado de secundaria sobre las aspiraciones de progenitores y profesorado}

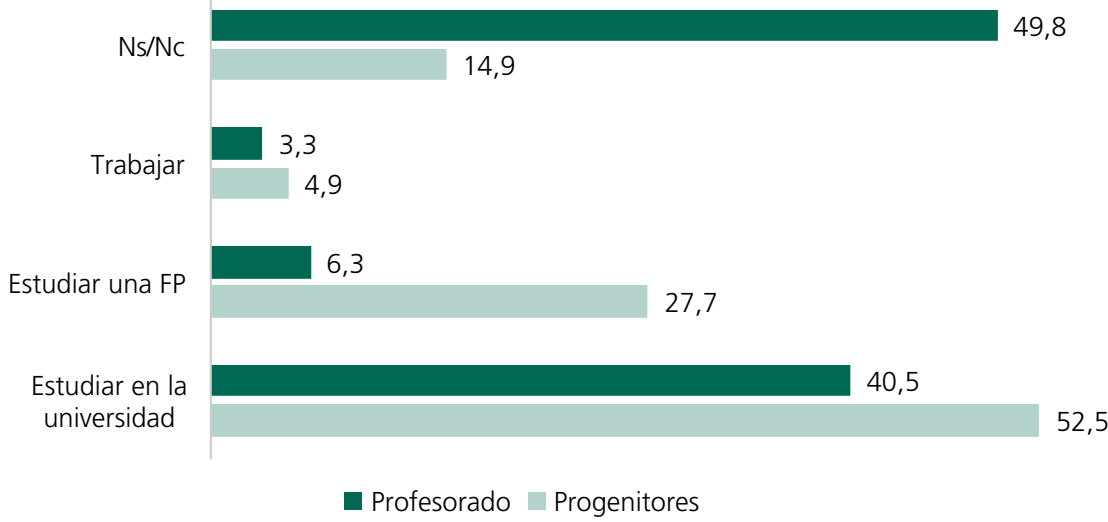

Fuente: Elaboración propia. P56. Y tus padres, ¿qué quieren que hagas después de acabar el instituto? P57. Y tus profesores/as ¿qué quieren que hagas después de acabar el instituto?

De nuevo, el sexo es una variable relevante para entender estas aspiraciones proyectadas. Un $43,5 \%$ de los chicos piensa que sus progenitores creen que acabará estudiando una formación profesional, diferenciándose significativamente de las chicas (7,5\%). Otra cuestión destacable es la formación de los progenitores. El alumnado con progenitores que tienen estudios superiores considera que en ningún caso su familia piensa que acabará estudiando una formación profesional, en comparación con aquellos que tienen estudios secundarios y de formación profesional $(40,9 \%)$ y primarios o sin estudios (15,3\%).

Es relevante también el contraste entre deseo y expectativa. Al preguntar al alumnado de ascendencia extranjera de secundaria en qué desea trabajar, y posteriormente dónde realmente se ubica dentro del mercado laboral, destaca sobre todo las dudas y el desconocimiento, ya que si bien un 6,3\% del alumnado nos indicaba que no sabía en qué le gustaría trabajar, una vez que planteamos que sean realistas, el dato asciende a un $24,8 \%$. En el caso de los empleos expuestos en la tabla 4 , se percibe una leve diferencia entre el deseo y lo que pueda ocurrir realmente. 
Tabla 4

\section{Aspiraciones y expectativas del alumnado de secundaria sobre su futuro laboral}

\begin{tabular}{|c|c|c|}
\hline & Deseo & Siendo realista \\
\hline Mecánico/a & 21,3 & 16,3 \\
\hline Médico/a y sanitario/a & 14,0 & 11,5 \\
\hline Futbolista & 9,9 & 0,0 \\
\hline Peluquera/o & 8,5 & 6,6 \\
\hline No sé & 6,3 & 24,8 \\
\hline Abogada/o & 6,1 & 6,1 \\
\hline Profesor/a & 5,8 & 5,8 \\
\hline Derecho & 3,3 & 3,3 \\
\hline Artista & 3,3 & 0,0 \\
\hline Azafata/o & 3,3 & 0,0 \\
\hline Administrativo/a & 3,3 & 3,3 \\
\hline Soldador & 3,0 & 0,0 \\
\hline Piloto & 3,0 & 6,3 \\
\hline Arquitecto/a & 2,5 & 2,5 \\
\hline Ingeniero/a & 2,5 & 0,0 \\
\hline Farmacéutico/a & 1,9 & 1,9 \\
\hline Transportista & 1,9 & 1,9 \\
\hline Albañil & 0,0 & 3,3 \\
\hline Tecnología & 0,0 & 3,0 \\
\hline Educador/a & 0,0 & 3,3 \\
\hline
\end{tabular}

Fuente: Elaboración propia. P58. Cuando seas mayor, ¿de qué te gustaría trabajar? P59. Y siendo realista, ¿de qué crees que podrás trabajar? 
Al igual que en el caso del alumnado de origen extranjero de primaria, preguntamos a los progenitores sobre algunos temas relacionados con el futuro laboral y formativo de sus hijos e hijas. De nuevo, tratamos de comprender las aspiraciones de los y las progenitores para poder así comprender las de sus hijos e hijas. Como podemos observar en el gráfico 5, ocho de cada diez quieren que sus hijos e hijas cursen estudios universitarios, un 10\% desea que estudien una formación profesional y un 5\% que comiencen a trabajar. Sus afirmaciones se apoyan en razones tales como que desean que vivan mejor que ellos, que consigan un trabajo o porque simplemente saben lo que sus hijos e hijas desean del futuro y apoyan su decisión.

\section{Gráfico 5}

\section{Aspiraciones de los progenitores de secundaria después del instituto}

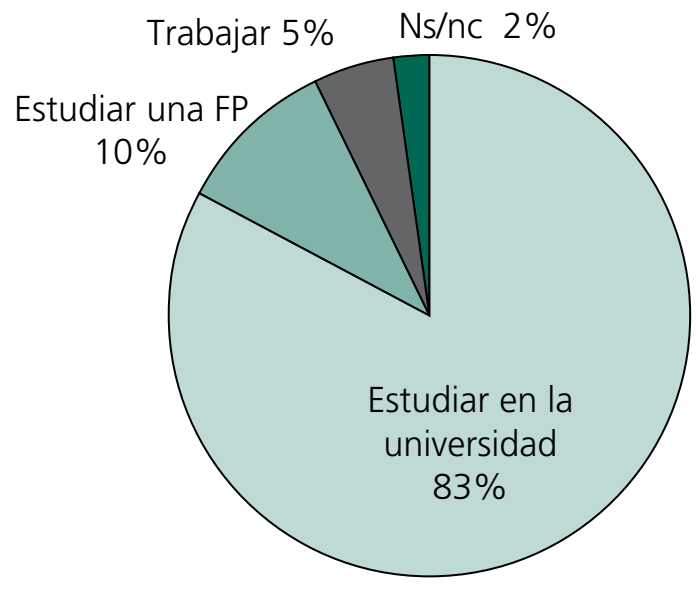

Fuente: Elaboración propia. P72. ¿Qué le gustaría que haga su hijo/a después de acabar el instituto?

Atendiendo a las diferencias existentes en estos deseos, de nuevo el modelo lingüístico es una variable relevante, ya que, el $50 \%$ de los progenitores que tiene a sus hijos e hijas en modelo $A$ cree que van a introducirse en el mercado laboral una vez finalicen el instituto, diferenciándose significativamente de quienes estudian en modelo $D$ (7\%). Asimismo, destacan dos cuestiones clave para comprender las expectativas maternas y/o paternas: el origen y el nivel de estudios. En relación a la primera, los progenitores de origen asiático desean 
por encima de la media que su descendencia se incorpore al mercado laboral tras completar la educación obligatoria (25\%), mientras que el $86 \%$ de los padres y/o madres de origen latinoamericano desean que su descendencia vaya a la universidad, también por encima de la media. Respecto al nivel de estudios de los progenitores, la influencia de esta variable en las aspiraciones de la descendencia es una cuestión ampliamente estudiada. En la siguiente tabla 5 observamos que el $62,6 \%$ de las personas sin estudios o estudios primarios desea que sus hijos/as acudan a la universidad, porcentaje muy inferior a la media (82,9\%). Muestran además una mayor indefinición o incertidumbre respecto al futuro de su descendencia, con un $12,6 \%$ de $« \mathrm{Ns} / \mathrm{Nc}$.

\section{Tabla 5}

\section{Aspiraciones de los progenitores de secundaria sobre} el futuro académico de sus hijos e hijas

\begin{tabular}{|l|c|c|c|c|}
\hline & Total & $\begin{array}{c}\text { Sin estudios/ } \\
\text { Primarios }\end{array}$ & Secundaria/ FP & Superiores \\
\hline Estudiar en la universidad & 82,9 & 62,6 & 89,5 & 83,5 \\
\hline Estudiar una FP & 9,6 & 15,3 & 5,5 & 16,5 \\
\hline Trabajar & 4,9 & 9,5 & 5,0 & 0,1 \\
\hline Ns/nc & 2,5 & 12,6 & 0,0 & 0,0 \\
\hline
\end{tabular}

Fuente: Elaboración propia. P72. ¿Qué le gustaría que haga su hijo/a después de acabar el instituto?

Al igual que preguntábamos anteriormente al alumnado de origen extranjero de secundaria sobre en qué le gustaría trabajar y realmente dónde se ve trabajando, hacemos las mismas dos preguntas a sus progenitores. Como podemos observar en la tabla 6 , las aspiraciones de las familias de que estudien lo que a ellos les guste pasa de un $31,1 \%$ a un $5,8 \%$ cuando piensan realmente en qué van a trabajar. Las dudas aumentan, sin embargo, cuando titubean sobre el trabajo que desempeñarán en un futuro $(46,6 \%)$. 
Tabla 6

\section{Aspiraciones y expectativas de los progenitores de secundaria sobre el futuro profesional de sus hijos e hijas}

\begin{tabular}{|c|c|c|}
\hline & Deseo & Siendo realista \\
\hline Lo que le guste/quiera & 33,1 & 5,8 \\
\hline Profesora & 6,6 & 6,6 \\
\hline Médico & 7,4 & 8,2 \\
\hline Ingeniero & 5,5 & - \\
\hline Informática & 3,0 & 6,1 \\
\hline Administrativo & 6,3 & - \\
\hline Comunicación & 3,3 & - \\
\hline Buen puesto & 3,3 & - \\
\hline Arquitecto & 1,9 & 2,5 \\
\hline En lo que pueda & 3,0 & 3,3 \\
\hline Abogada & 6,3 & 6,3 \\
\hline Piloto & 3,0 & 3,0 \\
\hline Peluquera & 1,9 & - \\
\hline Farmacéutico & 1,9 & - \\
\hline No sé & 13,3 & 46,6 \\
\hline Mecánico & 一 & 3,3 \\
\hline Empresario & - & 1,9 \\
\hline Soldador & - & 3,0 \\
\hline
\end{tabular}

Fuente: Elaboración propia. P74. ¿De qué le gustaría que trabaje su hijo/s de mayor? P75. Y siendo realista, ¿de qué cree que podrá trabajar?

Ante el resto de profesiones, no percibimos excesivas diferencias entre las aspiraciones y expectativas, teniendo en cuenta que hablamos de alumnado de secundaria donde sus familias pueden tener algo más claro cuáles pueden ser sus preferencias, no como ocurría con el alumnado de primaria, donde podía entreverse más claramente los deseos de 
unos progenitores ante un futuro mucho más lejano que con el que nos encontramos ahora.

\subsubsection{EXPECTATIVAS MIGRATORIAS}

Las expectativas migratorias de la familia muestran la intención de continuar en el territorio o de desplazarse a otro (origen o una nueva migración) y son además un indicador indirecto de satisfacción o insatisfacción con su situación actual y su confianza en el futuro. Así, el análisis de la pregunta sobre su futuro de aquí a cinco años — si tienen pensado seguir residiendo en el País Vasco, marcharse a otro país o comunidad autónoma o regresar a origen- nos muestra en el gráfico 6 que las familias de origen extranjero tienen pensado quedarse en el País Vasco mayoritariamente $(87,1 \%)$, mientras que el resto de las opciones son residuales.

\section{Gráfico 6}

\section{Expectativas de aquí a cinco años}

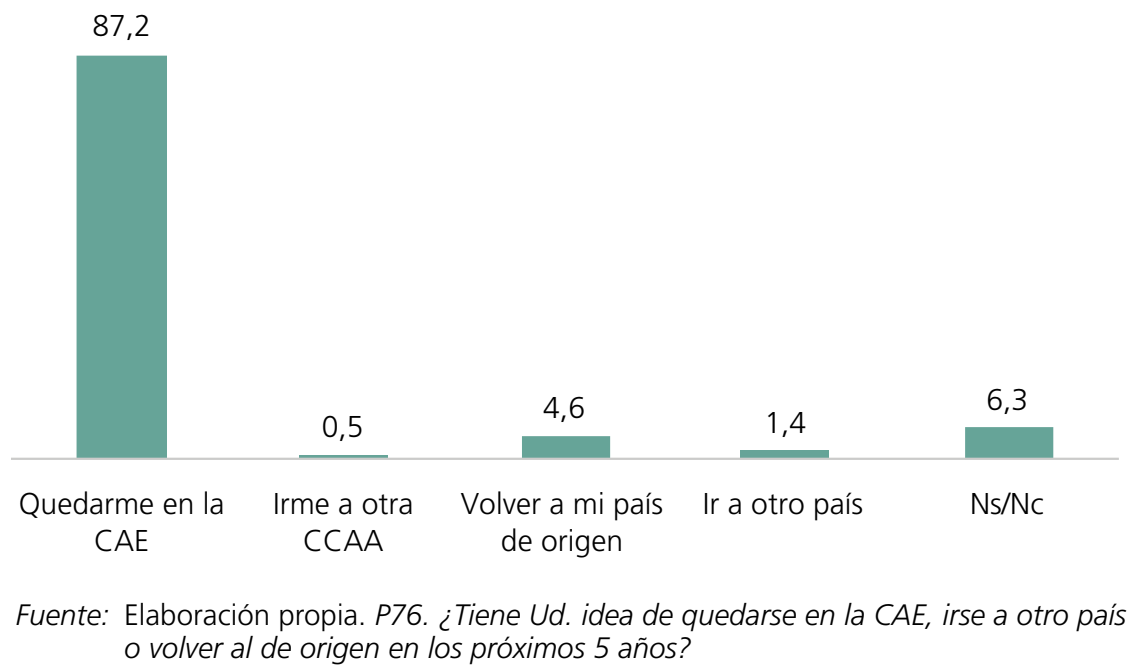

Tan solo un 4,6\% tiene intención de volver a su país de origen y un $1,4 \%$ de marcharse a otro país. Un $9,5 \%$ de la población de origen extranjero que llegó al territorio en el último periodo comprendido entre 2008 y 2013 piensa irse a otro país, diferenciándose significativamente de periodos anteriores. 


\subsection{Expectativas y aspiraciones desde la perspectiva de las familias}

Tras conocer el volumen y tendencias de las aspiraciones y las expectativas, se analizan los discursos en torno a estas, fruto de las entrevistas a unidades familiares de diversos orígenes extranjeros. En este apartado, se analizarán no solo las expectativas y las aspiraciones que unos y otros manifiestan, también cuáles son, en su opinión, las cuestiones clave para lograr ese futuro optimista que padres y madres proyectan para su descendencia, pero en menor medida para sí mismos ${ }^{1}$.

\subsubsection{INTEGRACIÓN Y EDUCACIÓN}

Cabe destacar, como paso previo al análisis de las expectativas, dos cuestiones estrechamente relacionadas y que el análisis cuantitativo y la literatura revelan como claves: la interpretación de las familias sobre la integración y el interés de los progenitores sobre la educación.

En primer lugar, se analiza la actitud de los progenitores respecto a la educación. El estudio, de corte cuantitativo, sobre la diversidad en las aulas (Fernández, Shershneva y Fouassier 2017), mostraba que el alumnado de ascendencia extranjera requería de más ayuda con los deberes que el alumnado de familias de origen autóctono. En aquellas familias donde los progenitores tienen estudios de primaria o no tienen estudios hay más casos de niños y niñas a los que no se les ayuda con los deberes, situación que se da en mayor medida entre las familias de ascendencia extranjera. Es importante subrayar este hecho para matizar el discurso de la educación pública como igualador social o motor de la movilidad social. La formación y el rendimiento académico se desarrollan fundamentalmente en el centro escolar, pero también en el hogar, y es en ese punto donde datos y discursos nos muestran desigualdades y dificultades para las familias de ascendencia extranjera. En el análisis cualitativo, se hace evidente esta dificultad para ayudar con los deberes, que recae en mayor medida sobre las madres o mujeres de la familia. De hecho, entre las tareas reproductivas, la atención a las y los niños recae especialmente sobre las madres, que afirman ser las encargadas en muchos casos de los temas escolares como las reuniones con el profesorado, los deberes, la participación en AMPAs o la agenda escolar.

1 Al tratarse de un artículo con una extensión limitada, se incluyen exclusivamente algunas citas que ilustren lo mencionado; en ningún caso representan el total de las citas recogidas, son tan solo un ejemplo. 

ellos?

¿Tienes buena relación con los profesores? ¿Hablas mucho con

M: Sí, casi sólo hablo yo. Porque como estoy más cerca... pero ha sido así de siempre.

Siempre te has encargado tú, siempre has sido tú la persona de referencia.

M: Sí. (Rumanía, monoparental, Álava)

Respecto a la integración de adultos y niños y niñas, los discursos vinculan las relaciones sociales de las familias de ascendencia extranjera a su proceso de integración ya que, como ellas mismas afirman, existen diferencias entre los círculos de los progenitores y su descendencia. Entre los primeros, las redes de amistades y apoyo son más homogéneas - con mayor presencia de personas de origen extranjero- mientras que entre los segundos la diversidad escolar y social se manifiesta en redes muy heterogéneas. En los discursos analizados la integración de progenitores y descendientes aparecía vinculada a la educación, pero, especialmente, a las relaciones sociales. Los progenitores nos hablan de las relaciones y círculos de amistades casi en términos de capital cultural y social (Bourdieu 1977), asumiendo que un círculo formado por personas «de aquíl» garantiza una inmersión cultural y lingüística y que dota al niño o niña de una red de apoyo. Una red que puede llegar incluso a procurar empleo en un mercado laboral basado en «los contactos». Una definición de integración de corte muy culturalista y de carácter asimilacionista, ya que no solo es necesario conocer la cultura local, sino adoptarla. Seguramente, un reflejo de la opinión mayoritaria de la sociedad vasca que, de hecho, concibe la integración, mayoritariamente, desde la asimilación (Ikuspegi 2019).

La integración que tú preguntas es eso, conocer lo que pasa aquí y conocer a gente de aquí. Yo casi no conozco gente de aquí, pero ellos sí. Yo solo por el colegio. Por eso igual uno le va a decir que vaya a trabajar a su empresa cuando sea mayor. Eso es la integración, los amigos de aquí.

¿Entonces, ellos van a estar más integrados que tú?

Sí, porque ellos ya son de aquí, pero no por nacer aquí, porque tienen la cultura de aquí, hablan como se habla aquí y sus amigos son de aquí. Yo la cultura la tengo de allá y aquí mis amigos también son de allá (América Latina, monoparental, Gipuzkoa) 


\subsubsection{EXPECTATIVAS}

En relación a las expectativas, de manera espontánea los progenitores mencionaban la educación o formación de sus hijos e hijas. Las expectativas de padres y madres deben analizarse en profundidad como medio para comprender las de sus hijos e hijas, ya que, como se menciona en el marco teórico, la actitud hacia la formación y expectativas de los progenitores influye en el rendimiento y aspiraciones de sus descendientes a través del proceso de socialización (GonzálezPineda, et al. 1997). En este sentido, la universidad es el objetivo de casi todos los progenitores para su descendencia en los discursos analizados, entienden la educación superior como medio para acceder a un buen empleo, como ya mencionábamos, la base sobre la que construir un futuro mejor. Ya se observaba en el apartado cuantitativo que la universidad es una prioridad para el $82 \%$ de los progenitores, mostrando una evidente coherencia con los discursos encontrados. A la formación universitaria le acompaña el nivel adecuado de euskera, asumiendo el castellano como lengua completamente dominada y que, de algún modo, no diferencia en lo social o lo laboral.

\section{¿Qué es lo que te gustaría estudiar?}

Ho1: Pues ingeniero informático.

Muy bien. ¿Y tú?

Ho2: Yo no lo he pensado todavía.

¿Y a ti qué te gustaría?

Ma: Lo que quieran ellos. Cuando le dices una cosa, por ejemplo, médico, si a él no le gusta, va al cole con asco. Así que algo que le guste.

¿Pero qué nivel de estudios te gustaría que hicieran? Si tienes alguna expectativa o alguna idea.

Ma: Sí, yo pienso que vayan a la universidad los dos. Ir a la universidad para tener un trabajo muy bueno después, para no trabajar como yo y como su padre. Pero ahora depende todo de ellos. (Rumanía, monoparental, Álava)

La dupla formación universitaria y euskera se revela en casi todos los discursos como la forma en que sus descendientes alcanzarán el éxito, aunque no lo garantiza ya que «también depende de la suerte» y «de cuánta gente conoces».

Los discursos muestran un nivel más moderado y superficial, donde los y las progenitores afirman no tener inclinación alguna respecto a los estudios que cursen sus hijos e hijas; y otro nivel de carácter más directo y profundo, en el que padres y madres manifiestan sus preferencias. Estas se centran en los estudios universitarios, llegando incluso a especificar 
el tipo de grado (medicina parece la preferida en gran cantidad de discursos). En todo momento las expectativas educativas se unen a las laborales, apareciendo el empleo público como la opción más interesante para sus hijos y/o hijas. En el apartado cuantitativo observábamos ya que profesiones como medicina o policía aparecían en los primeros puestos del ranking de padres y madres.

¿Qué te gustaría que hiciera ella en el futuro?

Ha: Pues tener buen futuro, me imagino. Estudiar y sacar una carrera...

No sé, alguna cosa en la que te gustaría que trabajara tu hija. Algo que suelas decirle...

Ha: Pues a mí me gustaría que trabajara de médico o por lo menos enfermera. Si no puede, de auxiliar de enfermera. Que no esté, como yo, limpiando váteres, limpiando baños. Voy a intentar darle lo mejor y exigirla y ayudarla para por lo menos sacar buenos estudios y tener un buen trabajo. Y para un buen trabajo, que trabaje en el Estado.

$Y a$, de funcionaria con una plaza fija.

Ha: Eso es, con una plaza fija. O de policía si hace falta. Mira, ella tiene fobia de la Policía... (Magreb, extensa, Álava)

Sin embargo, los hijos y las hijas no siempre están de acuerdo con la perspectiva paterna y/o materna. La propia dinámica generada en el contexto del microgrupo resultaba en interesantes debates entre progenitores y descendientes. Las aspiraciones de carácter más artístico (música o pintura, por ejemplo), que se perciben con pocas salidas profesionales o que no contemplan la universidad como parte de su camino, generan cierto rechazo y tensión en la conversación.

M: A mí me gustaría que se dejara de música y de móvil y de jugar a máquina y fuera a vuestra universidad, pero no dice nada ni mira nada... mira, no quiere ni oír el tema, se va. (América Latina, monoparental, Gipuzkoa)

Las proyecciones acerca del futuro mostraban en los discursos dos perspectivas muy diferenciadas: una de carácter más pesimista y otras más optimista. Sin embargo, los testimonios de padres y madres, de forma general, presentan un carácter algo pesimista respecto a su devenir, que pasará necesariamente por el empleo, ya sea mantenerlo, encontrarlo o mejorar sus condiciones.

No obstante, el discurso se tornaba mucho más optimista al plantear a los progenitores el futuro de su descendencia. En opinión de los padres y madres, la mejor posición estratégica de su descendencia tendrá como consecuencia la posibilidad de tener una vida mejor que la de 
sus progenitores. Con incertidumbres, por supuesto, pero con muchas posibilidades de éxito —en muchos discursos, vinculadas al trabajo-.

Entonces, te imaginas un futuro bueno para ellos, ¿no?

Si se quedan aquí sí. El futuro de los hijos va a estar bien. Ellos van a saber euskera, van a tener a sus amigos y a mí. Ellos van a ser de aquí y les va a ir como a los de aquí.

¿Cómo a un vasco?

Sí, igual de bien. O de mal. (América Latina, monoparental, Gipuzkoa)

Pueden extraerse de lo anteriormente expuesto dos posturas con diferentes objetivos: la de la descendencia, basada en encontrar una trayectoria que les permita vivir económicamente bien, pero a la vez les genere interés y realización; y otra, la de padres y madres, con un objetivo claro: la movilidad social ascendente. Como se ilustra en el gráfico 7, una movilidad basada en la integración laboral y la integración social de las hijas e hijos de ascendencia extranjera.

\section{Gráfico 7}

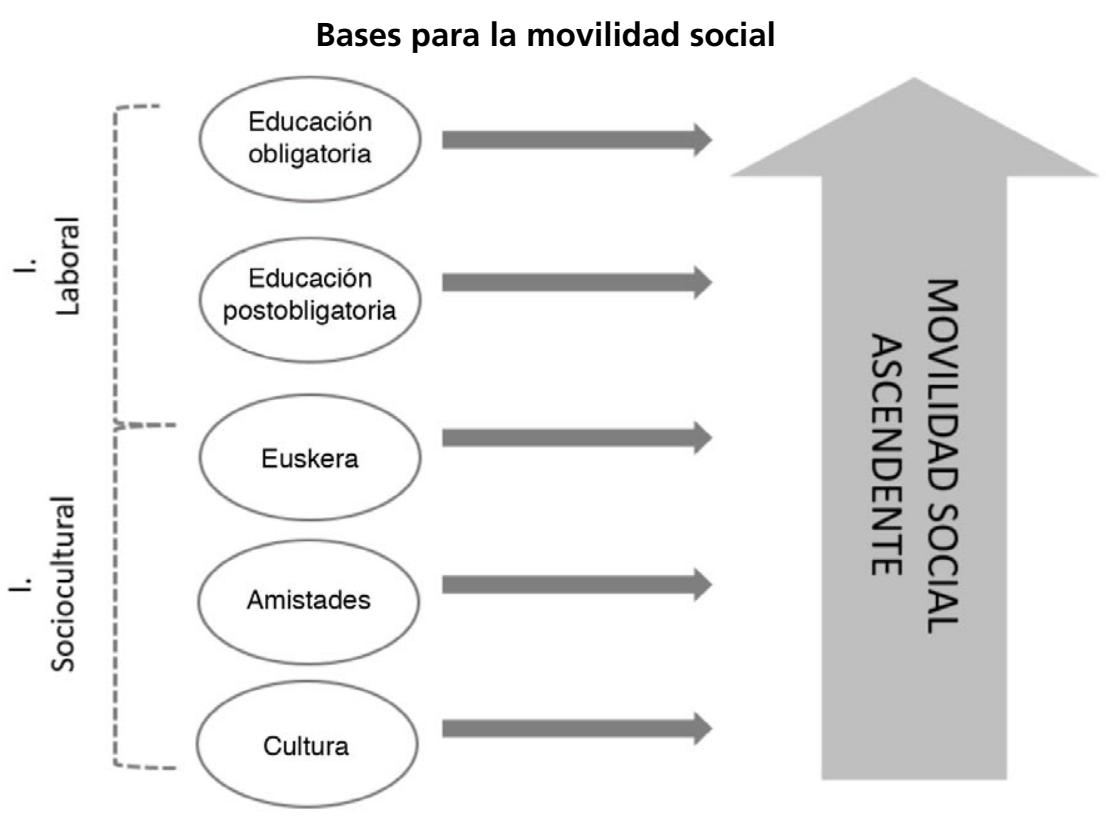

Fuente: Elaboración propia. 
Es decir, el objetivo de los progenitores es que sus hijos e hijas logren una movilidad social ascendente a través de la suma de la educación obligatoria, la universitaria y el conocimiento de euskera. A esta fórmula se le sumaría la integración social, como ya mencionábamos, entendida como el dominio de los idiomas locales, la cultura vasca y las amistades con personas de origen autóctono, fruto de la socialización en el País Vasco. La suma de ambos bloques daría como resultado una «igualdad de condiciones» que, en los discursos analizados, generará la diferencia en el futuro de sus hijos e hijas o, dicho de otro modo, diferenciará a las personas inmigrantes de las de ascendencia extranjera procurándoles un futuro mejor.

Sin embargo, no debemos perder de vista que, tal y como mencionábamos en el marco teórico, la escuela y la familia son los elementos que forman en mayor medida el status y, en consecuencia, pueden ayudar a la movilidad social o generar una mayor desigualad en las personas descendientes (Gil del Pino y García Segura 2019). En otras palabras, la fórmula para el ascenso social mencionada en los discursos analizados sería válida en un contexto meritocrático, sin embargo, las desigualdades de base en el núcleo familiar nos hacen sospechar que, solo con intervención pública, refuerzo del sistema educativo y apoyo institucional podrá garantizarse el ansiado ascenso social en igualdad de condiciones.

\section{Conclusiones}

El estudio de la descendencia de las personas de origen extranjero es un tema de gran complejidad, ya que requiere tener en cuenta varios niveles de análisis y variables que influyen en sus vivencias y aspiraciones. Además, una de las dificultades añadidas reside en el hecho de que este fenómeno tiene muy poco recorrido en el País Vasco, lo que implica construir un marco de análisis nuevo que contemple no solo su condición de ascendencia extranjera que, en muchos casos, marca su presente y futuro, sino también una serie de variables de contexto vasco, como el euskera o la cultura e identidad vasca, que complejizan aún más su estudio.

En este artículo se recogen los resultados de un primer acercamiento a esta realidad, centrándose sobre todo en las expectativas y aspiraciones tanto de los propios hijos e hijas de personas de origen extranjero como de sus progenitores.

Para ello, se ha optado por una doble metodología para no solo cuantificar el fenómeno en cuestión sino también para poder indagar en las motivaciones y argumentaciones que hay detrás de estos números. 
En primer lugar, cabe destacar que las familias de origen extranjero, al igual que las autóctonas, son conscientes de la importancia de la educación en el logro de una movilidad social ascendente. En este sentido, los y las jóvenes de ascendencia extranjera desean acceder a la educación superior, muy por encima de la formación profesional. Al mismo tiempo, la entrada directa al mercado laboral es muy poco frecuente, siendo este porcentaje más alto en el caso de personas de origen asiático. Esta misma tendencia se observa cuando se les pregunta a sus progenitores, que también desean que sus descendientes estudien en la universidad como primera opción, mientras que solo uno de cada diez opta por la formación profesional. En este sentido, el análisis cualitativo confirma esta preferencia por seguir estudiando después de la educación secundaria obligatoria, y especialmente por la educación superior. Así, la educación superior es entendida como uno de los factores que pueda proporcionarles a los descendientes de las personas de origen extranjero un futuro más próspero y estable, siendo una condición básica para una movilidad social ascendente. En esta misma línea, los estudios previos hablan de la importancia de las estrategias familiares (Garreta 1994, Portes et al. 2011), siendo estas uno de los factores que determinan las aspiraciones de los y las jóvenes.

Sin embargo, la población encuestada no es homogénea, con lo que observamos diferencias significativas en algunas variables sociodemográficas. Observamos, por tanto, que la universidad como meta educativa es más común en el caso de los padres y/o madres con estudios superiores o medios. Este dato es congruente con los resultados de otros estudios que afirmaban que las familias de nivel socioeconómico más elevado tienden a tener mayores expectativas educativas de sus descendientes (Lozano y Trinidad 2019).

El sexo es otra variable que marca la diferencia en las respuestas obtenidas: hay más chicas que optan por la universidad que chicos, en cuyo caso la formación profesional es algo más frecuente. En esta misma línea, el estudio de Portes et al. (2011) apuntaba que las chicas presentan una mayor ambición educativa que los chicos.

Por otro lado, es interesante el contraste que hacen los hijos e hijas de ascendencia extranjera con lo que piensan que esperan de ellos y ellas sus progenitores y el profesorado. Cabe señalar que las expectativas de estos agentes de socialización más importantes es una cuestión esencial en la formación de aspiraciones hacia el futuro y el rendimiento escolar (Van Houtte 2011, Carrasco, Pàmies y Narciso 2018). En el caso de estos jóvenes, vemos que perciben que sus progenitores esperan que estudien en la universidad en mayor medida 
que sus profesores y profesoras, aunque también es cierto que el desconocimiento de las expectativas del profesorado acerca de sus futuros estudios es muy alto.

Por otro lado, nos hemos interesado por las profesiones que les gustaría desempeñar de mayor a las y los hijos de las personas de origen extranjero. En este caso, es importante diferenciar entre el deseo y la creencia de lo que pueda ocurrir en la realidad. De entre las opciones sugeridas, medicina es la opción más mencionada tanto por los progenitores como por los propios jóvenes. Sin embargo, cuando se les pregunta qué creen que van a ser en el futuro, los porcentajes descienden. Si bien los progenitores desean que sus hijos e hijas trabajen de lo que les guste, siendo realistas se inclinan por opciones «más prácticas» desde su punto de vista. Este razonamiento también aparece en las entrevistas familiares donde los progenitores se inclinan por profesiones más estratégicas desde el punto de vista de la movilidad social y un futuro próspero económicamente, mientras que sus hijos e hijas aspiran a poder trabajar no solo de lo que les aporte esta estabilidad sino algo que también les guste y sea atractivo.

Aquí nuevamente aparece la importancia del capital cultural y educativo para una mayor integración social y económica que buscan los padres y madres para sus descendientes. En este sentido, el discurso de las familias pone sobre la mesa la importancia de la educación, junto con el dominio del euskera y una red de amistades autóctonas que interpretan como una fuente de integración y ascenso social.

Por último, es interesante la proyección que hacen las personas de origen extranjero del futuro de sus descendientes. La mayoría se muestra muy optimista y considera que sus hijos e hijas van a lograr una mayor estabilidad y éxito laboral y social de la que ellos van a llegar a disfrutar, puesto que consideran que no van a tener que enfrentarse a los mismos obstáculos que se derivan de la condición de inmigrante.

\section{Referencias bibliográficas}

Alcalde, R. y R. Hernández. 2018. «Estilos motivacionales y expectativas educativas en las familias inmigrantes: un análisis basado en el estudio de casos de familias inmigrantes de origen dominicano». Revista Complutense de Educación 29 n. ${ }^{\circ}$ 2: 455-474.

Aparicio, R. y A. Tornos. 2006. Los hijos de inmigrantes que se hacen adultos: marroquies, dominicanos, peruanos. Documentos del Observatorio Permanente de la Inmigración, 8. Madrid: Ministerio de Trabajo y Asuntos Sociales. 
Aparicio, R. y A. Portes. 2014. Crecer en España. La integración de los hijos de inmigrantes. Colección de Estudios sociales, 38. Barcelona: Obra Social La Caixa.

Barquín, A. 2007. «Relación entre culturas y desarrollo comunitario: la oportunidad de conciliar igualdad y diversidad en la escuela». En Inmigración y culturas minorizadas: políticas sociales y criminales, coordinado por C. Manzanos, 105-114, Vitoria-Gasteiz: Ikusbide.

Barquín, A. 2015. "¿Qué debe hacer la escuela con las culturas familiares del alumnado inmigrante?». Educar 51, n. ${ }^{\circ}$ 2: 443-464.

Bourdieu, P. 1977. Capital cultural, Escuela y Espacio Social. Madrid: Siglo XXI.

Bourdieu, P. 1979. «Los tres estados del capital cultural». Sociológica 2, n. ${ }^{\circ}$ : 11-17.

Bourdieu, P. 1986. «The forms of capital». En Handbook of Theory and research for the Sociology of Education, editado por J.G. Richardson, 241-258. New York: Greenwood.

Carrasco, S., J. Pàmies y M. Bertran. 2009. «Familias inmigrantes y escuela: desencuentros, estrategias y capital social». Revista Complutense de Educación 20, n. ${ }^{\circ}$ 1: 55-78.

Carrasco, S., L. Narciso y M. Bertran-Tarrés. 2018. «Neglected aspirations. Academic trajectories and the risk of Early School Leaving among immigrant and Roma youth in Spain». En Comparative Perspectives on Early School Leaving in the European Union. Taking a multi-methods approach towards a multi-level social phenomenon, editado por Van Praag et al., 164-182. Londres: Routledge.

Carrasco, S., J. Pàmies, y L. Narciso. 2018. "Abandono escolar prematuro y alumnado de origen extranjero en España: ¿un problema invisible?». Anuario CIDOB de la Inmigración, 212-237.

Cebolla, H. y A. Martínez de Lizarrondo. 2015. "Las expectativas educativas de la población inmigrante en Navarra ¿optimismo inmigrante o efectos de escuela?». Revista Internacional de Sociología 73, n. ${ }^{\circ}$ 1. http://dx.doi. org/103989/ris.2013.02.22

Checa, J.C. y A. Arjona. 2009. «La integración de los inmigrantes de «segunda generación» en Almería. Un caso de pluralismo fragmentado». Revista Internacional de Sociología 67, n. ${ }^{\circ}$ 3: 701-727.

Coleman, J.S. 1968. "The concept of equality of educational opportunity». Harvard Educational Review 38: 7-37.

Etxeberria, F. y M.C. Elosegui. 2010a. «Alumnado inmigrante: entre la asimilación y la marginación». SL\&i en red: segundas Lenguas e inmigración en red, 4: 21-41.

Etxeberria, F. y M.C. Elosegui. 2010b. «Integración del alumnado inmigrante: obstáculos y propuestas». Revista Española de Educación Comparada 16: 235-263.

Fernández, I., J. Shershneva y M. Fouassier. 2017. «La diversidad en las aulas: perspectiva cuantitativa». En La diversidad infantil y juvenil en la CAE. Las (mal) llamadas segundas generaciones, editado por J.A. Oleaga, 337-500. Bilbao: UPV/EHU. 
Garreta, J. 1994. "Expectativas educativas y sociales de las familias inmigrantes». Papers 43: 115-122.

Gil del Pino, C. y S. García Segura. 2019. «Influencia de las expectativas de familias y profesorado en el logro educativo y social de los estudiantes». Educaçao e Pesquisa Revista da Faculdade de Educação da Universidade de São Paulo 45. https://doi.org/10.1590/s1678-4634201945214529

González-Pineda, J.A, J.C. Núñez, S. González-Pumariega et al. 1997. «Autoconcepto, autoestima y aprendizaje escolar». Psicothema 9, n. ${ }^{\circ} 2$ : 271-289.

Gualda, E. 2010. La segunda generación de inmigrantes en Huelva: estudio HIJAI. El Masnou: Diálogos-Red.

Hanifan, L.J. 1916. "The rural school community center». Annals of the American Academy of Political and Social Science 67: 130-138.

Ikuspegi. 2019. Barómetro sobre las percepciones y actitudes hacia la población de origen extranjero. Acceso el 23 de mayo de 2020. https:// www.ikuspegi.eus/documentos/barometros/2019/bar_CAE_2019_CAS. pdf

Intxausti, N., F. Etxeberria y L. Joaristi. 2014. «¿Coinciden las expectativas escolares de la familia y del profesorado acerca del alumnado de origen inmigrante?». Relieve 20, n. ${ }^{\circ}$ 1. DOI: 10.7203/relieve.20.1.3804

Kao, G. y M. Tienda. 1998. «Educational aspirations of minority youth». American Journal of Education 106: 349-384.

Lizasoain, L., L. Joaristi, J.F. Lukas y K. Santiago. 2007. «El efecto contextual del nivel socioeconómico sobre el rendimiento académico en la educación secundaria obligatoria en la Comunidad Autónoma Vasca (España). Estudio diferencial del nivel socioeconómico familiar y del centro escolar». Archivos Analíticos de Políticas Educativas 15, n. ${ }^{\circ}$ 8: 1-37.

Lozano, M.A. y A. Trinidad. 2019. «El capital cultural como predictor del rendimiento escolar en España». International Journal of Sociology of Education 8, n. ${ }^{\circ}$ 1: 45-74.

Majoribanks, K. 2003. "Family background, individual and environmental influences, aspirations and young adults' educational attainment: a follow-up study». Educational Studies 29, n. ${ }^{\circ} 2$ : 233-242.

Oleaga, J.A. ed. 2017. La diversidad infantil y juvenil en la CAE. Las (mal) Ilamadas segundas generaciones. Bilbao: UPV/EHU.

Otero, B. y K. Mendoza. 2017. "La diversidad infantil y juvenil de la CAE en cifras». En La diversidad infantil y juvenil en la CAE. Las (mal) llamadas segundas generaciones, editado por J.A. Oleaga, 41-90. Bilbao: UPV/EHU.

Portes, A. y M. Zhou. 1993. "The new second generation: segmented assimilation and its variants». Annals of the American Academy of Political and Social Science 530: 74-96.

Portes, A. 1994. The new second generation. Nueva York: Russell Sage.

Portes, A. 2007. "Un diálogo Norte-Sur». En El país transnacional: migración mexicana y cambio social a través de la frontera, editado por M. Ariza y A. Portes, 651-702. México: UNAM, Instituto de Investigaciones Sociales. 
Portes, A., R. Aparicio y W. Haller. 2009. "La segunda generación en Barcelona: un estudio longitudinal». Análisis del Real Instituto Elcano 67. Acceso el 4 de marzo de 2020. http://www.realinstitutoelcano.org/

Portes, A., R. Aparicio, W. Haller y E. Vickstrom. 2011. «Progresar en Madrid: aspiraciones y expectativas de la segunda generación en España». REIS 134: 55-86.

Rumbaut, R.G. 1994. "The crucible within: ethnic identity, self-esteem, and segmented assimilation among children of immigrants». International Migration Review 28: 748-794.

Rumbaut, R.G. 2004. "Ages, life stages, and generational cohorts: decomposing the immigrant first and second generations in the United States». International Migration Review 38: 1160-1205.

Sewell, W.H., A.O. Haller y A. Portes. 1969. "The educational and early occupational attainment process». American Sociological Review 34: 82-92.

Torio, S., J. Hernández y J.V. Peña. 2007. «Capital social familiar y expectativas académico-formativas y laborales en el alumnado de Educación Secundaria Obligatoria». Revista de Educación 343: 559-586.

Torrents, D., R. Merino, M. García, y O. Valls. 2018. «El peso del origen social y del centro escolar en la desigualdad de resultados al final de la escuela obligatoria». Papers 103, n. ${ }^{\circ}$ 1: 29-50.

Van Houtte, M. 2011. "So, where's the teacher in school effects research? The impact of teachers, beliefs, culture and beaviour on equity and excellence in education». En Equity and excellence in education: towards maximal learning opportunities for all students, editado por K. Van Den Branden, G. Van Avermaet y M. Van Houtte, 75-95. Nueva York: Routledge.

Vicente Torrado, T.L. 2006. «Población inmigrante en los centros educativos vascos: valoración del personal docente». En Inmigración, miradas y reflejos: historias e identidades y claves de intervención social, editado por C. Maiztegui y R. Santibáñez, 127-150. Bilbao: Universidad de Deusto.

Vicente Torrado, T.L. 2008. La escuela vasca ante la realidad de la inmigración: un nuevo desafío. Vitoria-Gasteiz: Gobierno Vasco. 


\section{Copyright}

Deusto Journal of Human Rights / Revista Deusto de Derechos Humanos is an Open Access journal; which means that it is free for full and immediate access, reading, search, download, distribution, and reuse in any medium only for non-commercial purposes and in accordance with any applicable copyright legislation, without prior permission from the copyright holder (University of Deusto) or the author; provided the original work and publication source are properly cited (Issue number, year, pages and DOI if applicable) and any changes to the original are clearly indicated. Any other use of its content in any medium or format, now known or developed in the future, requires prior written permission of the copyright holder.

\section{Derechos de autoría}

Deusto Journal of Human Rights / Revista Deusto de Derechos Humanos es una revista de Acceso Abierto; lo que significa que es de libre acceso en su integridad inmediatamente después de la publicación de cada número. Se permite su lectura, la búsqueda, descarga, distribución y reutilización en cualquier tipo de soporte sólo para fines no comerciales y según lo previsto por la ley; sin la previa autorización de la Editorial (Universidad de Deusto) o la persona autora, siempre que la obra original sea debidamente citada (número, año, páginas y DOI si procede) y cualquier cambio en el original esté claramente indicado. Cualquier otro uso de su contenido en cualquier medio o formato, ahora conocido o desarrollado en el futuro, requiere el permiso previo por escrito de la persona titular de los derechos de autoría. 